OPEN ACCESS

Edited by:

Meng Zhou,

Wenzhou Medical University, China

Reviewed by:

Junfeng Xia,

Anhui University, China

Hongzhu Li,

Xiamen University, China

${ }^{*}$ Correspondence:

Wei Jiang

weijiang@nuaa.edu.cn

Lihong Wang

Iw2247@yeah.net

${ }^{t}$ These authors have contributed equally to this work

Specialty section:

This article was submitted to

Cancer Genetics,

a section of the journal

Frontiers in Oncology

Received: 28 October 2020 Accepted: 30 November 2020

Published: 14 January 2021

Citation:

Xing J, Liu H, Jiang W and Wang L (2021) LncRNA-Encoded Peptide: Functions and Predicting Methods.

Front. Oncol. 10:622294.

doi: 10.3389/fonc.2020.622294

\section{LncRNA-Encoded Peptide: Functions and Predicting Methods}

\author{
Jiani Xing ${ }^{1 \dagger}$, Haizhou $\mathrm{Liu}^{2 \dagger}$, Wei Jiang ${ }^{2 *}$ and Lihong Wang ${ }^{1,3^{*}}$ \\ ${ }^{1}$ Department of Pathophysiology, Medical College of Southeast University, Nanjing, China, ${ }^{2}$ Department of Biomedical \\ Engineering, Nanjing University of Aeronautics and Astronautics, Nanjing, China, ${ }^{3}$ Jiangsu Provincial Key Laboratory of \\ Critical Care Medicine, Nanjing, China
}

Long non-coding RNA (IncRNA) was originally defined as the representative of the noncoding RNAs and unable to encode. However, recent reports suggest that some IncRNAs actually contain open reading frames that encode peptides. These coding products play important roles in the pathogenesis of many diseases. Here, we summarize the regulatory pathways of mammalian IncRNA-encoded peptides in influencing muscle function, mRNA stability, gene expression, and so on. We also address the promoting and inhibiting functions of the peptides in different cancers and other diseases. Then we introduce the computational predicting methods and data resources to predict the coding ability of IncRNA. The intention of this review is to provide references for further coding research and contribute to reveal the potential prospects for targeted tumor therapy.

Keywords: long non-coding RNA, peptide, translation, cancer, Ribo-seq

\section{HIGHLIGHTS}

- We summarize the mechanisms of mammalian lncRNA-encoded peptides in regulating biological processes.

- We review the functions of IncRNA-encoded peptides in human diseases, especially in cancers.

- We sum up the classic computational methods and data resources for predicting lncRNAencoded peptides.

- This review may lay the foundation for further coding research and reveal the potential prospects for targeted tumor therapy.

\section{INTRODUCTION}

Non-coding RNA (ncRNA) is widely described as a class of RNA molecules transcribed from genomic DNA without coding capability (1). But with the improvements of bioinformatics and high-throughput technologies, open reading frames (ORFs) have been found in ncRNAs, such as pri-miRNA, circular RNA (circRNA), and long non-coding RNA (lncRNA) (2). These discoveries suggest that ncRNAs may encode proteins or peptides. The ORF of pri-miRNAs can encode peptides if they are directly transported to the cytosol without processing. For example, miPEP171b and miPEP165a, two peptides respectively encoded by pri-miR171b in Medicago truncatula and the pri-miR165a in Arabidopsis thaliana, can regulate root 
development by reducing expression of target genes (3). Besides ORFs, circRNA can be translated into proteins via internal ribosome entry site (IRES)-driven or $\mathrm{N}^{6}$-methyladenosine $\left(\mathrm{m}^{6} \mathrm{~A}\right)$-mediated initiation (4). Recently, a new coding type is reported in circRNA SHPRH which generates a protein from genetic codes overlapping. This protein protects itself from degradation by ubiquitination in gliomas $(5,6)$. Moreover, it has been found that a special circRNA is constituted by a head-to-tail circle form of IncRNA LINC-PINT exon 2, and encodes a peptide PINT87aa (7).

In addition to these ncRNAs, the regulatory mechanisms and functions of lncRNA coding products receive much concerns recently. LncRNAs are transcripts longer than 200nt and mainly located in the nucleus. The number of lncRNAs may exceed that of protein coding transcripts but its expression is generally lower. Owing to its abnormal expression and mutation, lncRNA is involved in many diseases, especially in cancers (8-10). Recent studies indicate that the translation of $\operatorname{lncRNAs}$ is driven by ORF, and some lncRNAs exert their functions through their coding peptides (2). For example, the 53 -amino acid (aa) peptide encoded by lncRNA HOXB-AS3 can inhibit colon cancer (11). LINC00689 encodes a 50aa peptide, which is highly similar to the signal recognition particle $19 \mathrm{kDa}$ protein (SRP19), and this coding ability was accelerated by the phosphorylated translation initiation factor eIF4E (12). Even though, it is rather difficult to evaluate the coding capacity of lncRNA due to high similarity to mRNA in structure, and sometimes the coding products are encoded in introns or overlapping exons of different genes. Only a minority of lncRNAs are functionally annotated at present, and there are still large amount of lncRNA-encoded products need to be discovered (13).

The coding products of lncRNAs has been studied in plants and invertebrates for a long time (14). As early as 2002, a soybean research discovered that a $679 \mathrm{nt} \operatorname{lncRNA}$, translated by early nodulin 40 gene, encoded two small peptides interacting with sucrose synthase (15). In addition, the lncRNA Toddler in zebrafish encodes a 58aa peptide and promoted gastrulation movements (16). But the study of IncRNA-encoded peptides in mammals is just beginning. Even though, there are still many exciting results achieved. Hitherto, some articles have introduced peptides encoded by lncRNAs in a wide range of species, and most of them are peptide-oriented (11, 17-19). However, in this review, we focused on the functions of mammalian lncRNA-encoded peptides oriented toward pathways and diseases, especially in tumors. We also included representative computational approaches and data resources for predicting the coding possibility.

\section{The Pathways Regulated by Long Non-Coding RNA-Encoded Peptides Inhibiting Mammalian Target of Rapamycin Complex 1}

The lysosome has the ability to degrade and recycle macromolecules, and its acidification is regulated by v-ATPase and the mammalian target of rapamycin complex 1 (mTORC1). V-ATPase activates mTORC1 and improves the excretion of essential amino acids from lysosomes (20, 21). LncRNA LINC00961 contains three
ORFs, one of which has been confirmed to encode the polypeptide SPAR by tandem mass spectrometry (MS). By specifically blocking vATPase, SPAR prevents mTORC1 activation that is stimulated by amino acids. Matsumoto et al. found that cell proliferation and muscle regeneration were accelerated when they knocked out the SPAR polypeptide in mice and injected a toxin into the muscle to induce injury (22), which suggested that SPAR was involved in the process of mTORC1 influencing muscle function (23). Besides, Spencer HL et al. found both LINC00961 and its coding peptide had independent function. LINC00961 itself acts as an angiogenesis inhibitor and interacts with actin-binding protein $\mathrm{T} \beta 4$ while its peptide promotes angiogenesis and binds to actin-binding protein SYNE1 (24). Moreover, a micropeptide LEMP encoded by lncRNA MyolncR4 also functions on muscles. It is shown that muscle development defect manifested in the reduction of muscle size and weight in LEMP KO mice. LEMP also promotes skeletal muscle activation and new fibers formation (25).

\section{Regulating Sarcoplasmic Reticulum $\mathrm{Ca}^{2+}$-ATPase}

The sarcoplasmic reticulum $\mathrm{Ca}^{2+}$-ATPase (SERCA) mediates muscle relaxation by pumping $\mathrm{Ca}^{2+}$ back into the sarcoplasmic reticulum, and its activity can be inhibited by sarcolipin (SLN) and phospholamban (PLN) (26). Some lncRNA-translated peptides have been reported as the regulators of SERCA. Skeletal musclespecific LINC00948 generates a micropeptide designated as myoregulin (MLN), which can form a transmembrane alphahelix. In addition, MLN has a similar hydrophobic binding motif as SLN and PLN for inhibiting the SERCA pump activity (27). In contrast, LOC100507537 encodes a 34aa peptide DWORF, which activates the SERCA pump. This DWORF peptide can counteract the SERCA inhibitor and reduce muscle contraction time (28). Furthermore, one lncRNA with two functional ORFs in Drosophila has been renamed as sarcolamban, which may have homology with SLN and PLN in vertebrates, as revealed through a cross-species sequence analysis (29).

\section{Participating in Messenger RNA Decay}

Non-sense-mediated mRNA decay (NMD) is the mRNA monitoring mechanism in eukaryotes, which decomposes mRNAs with premature termination codons. These mRNAs are produced during gene expression or cellular homeostasis maintenance processes (30). LINC01420, which is expressed only in mammalian cell lines, produces a polypeptide called NoBody. NoBody has neither homologous proteins nor a secondary structure, so its function can be measured only by proteomic techniques. In addition, the results suggest that NoBody is involved in mRNA decapping and degradation, which causes a decrease in cytoplasmic processing bodies (P-bodies) and a reduction in cellular NMD substrates (31). Recently, some studies have discovered that the NMD process may occur during the interaction between IncRNAs and ribosomes $(32,33)$. Another lncRNA, EPR, on the polyribosome is identified to have a 213 nucleotide-long ORF, which can recover mRNA stability by weakening the effect of the $\mathrm{KH}$-type splicing regulatory protein 
(KHSRP), while overexpressing the EPR-encoded polypeptide can promote epithelial cell tight junctions (34).

\section{Stimulating Mitochondria}

The mitochondrial inner membrane, which is the membrane unit wrapped around the mitochondrial matrix, contains a large quantity of cardiolipin and proteins. This membrane is responsible for metabolite transport, oxidative phosphorylation, ATP synthesis, and mitochondrial fission and fusion (35). Stein et al. found that LINC00116 could encode a 56aa microprotein called mitoregulin (Mtln), which localized to the mitochondrial inner membrane. Because Mtln forms a high molecular weight complex via the sticky intrinsically disordered protein regions (IDPR), it can strengthen the effect of the mitochondrial respiratory chain (MRC) and reactive oxygen species (36). Makarewich et al. demonstrated that the function of $1510011 \mathrm{k} 16 \mathrm{Rik}$ in mice was the same as its homolog LINC00116 in humans. In addition, 1510011k16Rik produces the micropeptide regulator of $\beta$-oxidation (MOXI). MOXI interacted with mitochondrial trifunctional protein to meet the energy requirements of an increasing metabolism and to control biological activity in other latent pathways (37). This discovery was subsequently confirmed by Chugunova et al. These authors suggested that MOXI can affect the activity of cytochrome b5 reductase 3 (Cyb5r3) associated with lipid metabolism and stimulate the MRC complex I (38).

In summary, lncRNA-encoded peptides can prevent mTORC1 activation to influence muscle function and regulate the SERCA pump activity to control muscle contraction time. Additionally, they can not only involve in mRNA stability and then mediate gene expression or cellular homeostasis maintenance processes, but also strengthen the MRC effect and ROS production. All above these were shown in Figure 1 and Table 1.

\section{The Functions of Long Non-Coding RNA-Encoded Peptides in Cancer}

Lately, peptides as tumor biomarkers have attracted increasing attention in clinical cancer treatments. Chakraborty et al. identified that the lncRNA translation products had a similar expression in 11 carcinoma cell lines, which showed great stability and succeeded as a general biomarker for cancer (50). Moreover, the coding peptides in other ncRNAs also function in malignant tumors. For example, a knockdown of microprotein CASIMO1, encoded by a ncRNA, can affect breast cancer cell proliferation. CircPPP1R12A encodes a 73aa protein, which helps colon cancer to metastasize (6, 51). Therefore, the lncRNA-encoded products are expected to be promising cancer targets and biomarkers in tumor therapies (18).

\section{Colon Cancer}

The peptide encoded by lncRNA HOXB-AS3 is involved in metabolic reprogramming to inhibit the growth of colon cancer. The ORF of HOXB-AS3 encodes a 53aa endogenous peptide. Kaplan-Meier analysis suggests that a lower expression of this 53 aa peptide is associated with a shortened survival time in colon

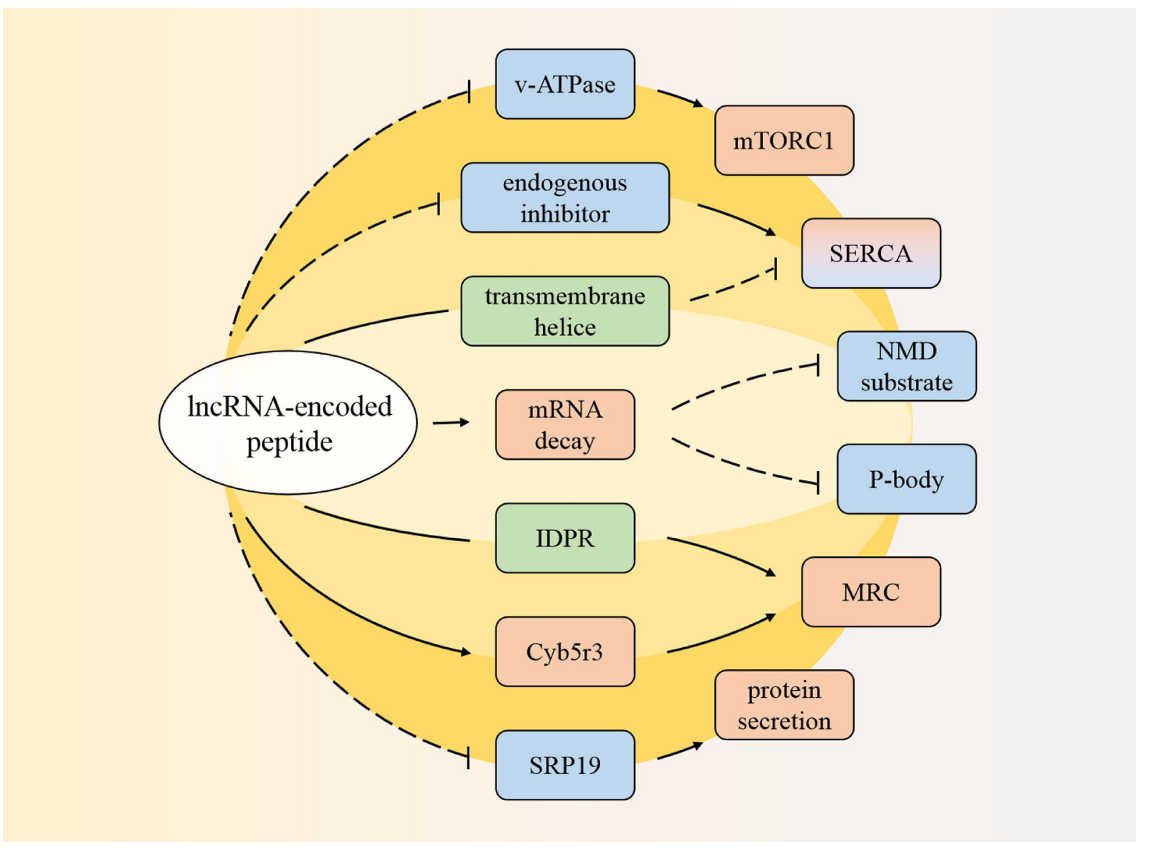

FIGURE 1 | Pathways regulated by the coding peptides. The peptide can prevent mammalian target of rapamycin complex 1 (mTORC1), and regulate sarcoplasmic reticulum $\mathrm{Ca}^{2+}$-ATPase (SERCA). One of them can also involve in messenger RNA (mRNA) decay to decrease cytoplasmic processing bodies (P-body) and cellular nonsense-mediated mRNA decay (NMD) substrate. In addition, one coding product has intrinsically disordered protein regions (IDPR), affects cytochrome b5 reductase 3 (Cyb5r3) to stimulate mitochondrial respiratory chain (MRC). Another coding peptide interferes signal recognition particle 19kDa protein (SRP19). Red: promotion; blue: inhibition; green: structure. 
TABLE 1 | The function of long non-coding RNA (IncRNA)-encoded peptides in mammals.

\begin{tabular}{|c|c|c|c|c|}
\hline LncRNA & Coding peptide & Length & Function of coding product & Reference \\
\hline LINC00689 & STORM & 50aa & Inhibit protein secretion. & $(12)$ \\
\hline \multirow[t]{2}{*}{ LINC00961/5430416009Rik (mouse) } & SPAR & 90aa & Inhibit mTORC1. & (22) \\
\hline & SPAAR & & Promote muscle development. & (24) \\
\hline MyolncR4 & LEMP & $56 a a$ & Promote muscle development. & (25) \\
\hline LINC00948/2310015B20Rik (mouse) & MLN & 46aa & Inhibit SERCA. & (27) \\
\hline NONMMUG026737 (mouse) & DWORF & 34aa & Activate SERCA. & (28) \\
\hline LINC01420 & NoBody & 63aа & Decrease P-bodies and mRNA. & (31) \\
\hline EPR (mouse) & EPRp & 71 aa & Promote epithelial tight junction. & (34) \\
\hline \multirow[t]{2}{*}{ LINC00116/1500011k16Rik (mouse) } & Mtln & $56 a a$ & Promote mitochondrion. & $(36-38)$ \\
\hline & MOXI (mouse) & & & \\
\hline HOXB-AS3 & - & 53aа & Suppress colon cancer. & (39) \\
\hline LOC90024 & SRSP & 130aа & Promote colon cancer. & $(40)$ \\
\hline LINC00266-1 & RBRP & 71 aa & Promote colon cancer. & $(41)$ \\
\hline LINC00998 & SMIM30 & 59aa & Promote liver cancer. & (42) \\
\hline HBVPTPAP & HBVPTPAP & $145 a a$ & Suppress liver cancer. & (43) \\
\hline LINC00278 & YY1BM & $21 a a$ & Suppress esophageal cancer. & $(44)$ \\
\hline LINC00665 & CIP2A-BP & $52 \mathrm{aa}$ & Suppress breast cancer. & (45) \\
\hline LINC00908 & ASRPS & 60aa & Suppress breast cancer. & $(46)$ \\
\hline meloe & MELOE-3 & 54aa & Possess immune tolerance. & $(47)$ \\
\hline MIR155HG & P155 & $17 \mathrm{aa}$ & Suppress autoimmune inflammation. & $(48)$ \\
\hline Aw112010 & - & 84aa & Cause immune response. & (49) \\
\hline
\end{tabular}

cancer patients. To distinguish whether the lncRNA or its peptide produced a degenerative effect in colon cancer, researchers mutated the start codon to delete the coding function of HOXB-AS3 and found that it was the coding peptide that influenced the formation of cancer cell colonies. This peptide decreases pyruvate kinase formation and reduces lactic acid production to inhibit colon cancer cell proliferation by antagonizing the splicing factor hnRNP A1 (39). Interestingly, the difference is that lncRNA HOXB-AS3 is downregulated in colon cancer but upregulated in acute myeloid leukemia. Papaioannou et al. deemed that the overlap of the lncRNA HOXB-AS3 transcript variants was limited in the two diseases. HOXB-AS3, which promotes the proliferation of acute white myeloid cells, is mainly located in the nucleus. It has a low correlation with polyribosome fragments, and there is no ribosomal protein enrichment in the eluate (52).

After confirming the anticancer activity of the lncRNA HOXBAS3-encoded peptide, Yan et al. recently provided other verification on a 130aa protein translated by lncRNA LOC90024 in advanced colorectal cancer. This $130 \mathrm{aa}$-protein was named splicing regulatory small protein (SRSP), due to its interaction with serine- and argininerich splicing factor 3 (SRSF3) to regulate mRNA splicing. The formation of the transcription factor long Sp4 isoform contains an adequate transactivating domain induced by SRSP binding to SRSF3 and regulates oncogene expression. Consequently, SRSF can promote colon cancer cell proliferation, migration, and invasion. In this example, alternative splicing provides an additional regulatory mechanism for transcription factor activity in cancer and leads to the activation of oncogenes and tumor progression (40).

Meanwhile, Yan et al. found 55 lncRNAs with different expression and coding potentials in the SW480 and SW620 cell lines and finally confirmed that among these, LINC00266-1 was able to produce RNA-binding regulatory peptide (RBRP). The $\mathrm{N}^{6}$ methyladenosine $\left(\mathrm{m}^{6} \mathrm{~A}\right)$ reader mediates $\mathrm{m}^{6} \mathrm{~A}$ recognition on RNAs, such as c-Myc mRNA. Thereby, RBRP, which binds to the $\mathrm{m}^{6} \mathrm{~A}$ reader IGF2BP1, can strengthen mRNA stability and increase the incidence of tumors. This $\mathrm{m}^{6} \mathrm{~A}$ recognition abnormality reveals a new way in which to target cancer (41).

\section{Liver Cancer}

An endogenous peptide SMIM30 has positive effect during the progression of hepatocellular carcinoma. Ribosomal protein S6 (RPS6) is the point to identify ORF existence. After being evaluated by software-coding potential calculator, LINC00998 sticks out from other RPS6-related lncRNAs and has high coding potential score. The study discovered that LINC00998 can produce a $7.3 \mathrm{kDa}$ peptide SMIM30 on cell membrane. If mutate the start codons of LINC00998's ORF, SMIM30 cannot perform its carcinogenesis by activating protein tyroaine kinase membrane anchoring and MAPK pathway (42).

It has been identified that lncRNA HBVPTPAP is overexpressed in the cytoplasm of HepG2 cell and inhibits cell proliferation significantly. There are two potential coding regions in HBVPTPAP, but one of them is excluded due to an overlap with the reference gene's exon. Another region can produce a polypeptide which interacts paired immunoglobulin like type 2 receptor alpha intracellular domain to activate JAK/STAT signaling pathway. Meanwhile, the peptide can also increase mitochondrial membrane potential and early apoptosis rate (43).

\section{Male Esophageal Squamous Cell Carcinoma}

Yin Yang 1-binding micropeptide (YY1BM) is a potential anticancer factor and can be encoded by the ORF in the first exon of LINC00278. The demethylated $\mathrm{m}^{6} \mathrm{~A}$ can improve this translation efficiency, which is mediated by the ALKBH5 protein induced by cigarette smoking. YY1BM can affect the binding between the transcription factor Yin Yang 1 and the androgen receptor to inhibit the transcription of eukaryotic elongation factor 2 kinase, which is the key factor in tumor adaptation to nutrient deprivation. A YY1BM knockout can reverse the nutrient deprivation-induced apoptosis in esophageal squamous carcinoma cells (44). 


\section{Breast Cancer}

In triple-negative breast cancer (TNBC), a micropeptide CIP2ABP translated from LINC00665 is found to inhibit the metastasis to lung. When Smad signal transduction pathway is stimulated by TGF- $\beta$ and then lead to the increasing combination between translation inhibitory protein 4E-BP1 and eIF4E, LINC00665's translation is suppressed and its coding peptide is reduced. Downregulated CIP2A-BP induces low activity of cancerous inhibitor of PP2A and promotes cancer metastasis and invasion in mouse mammary tumor model. While CIP2A-BP overexpression inhibits AKT phosphorylation and the TNBC progression (45).

At the same time, another peptide ASRPS produced by the third ORF of LINC00908 can inhibits TNBC. It is found that LINC00908 transcription is positively correlated with ER $\alpha$ and regulated directly by it, which cause the expression of ASRPS is low in TNBC. ASRPS can enhance the interaction between signal transducer and activator of transcription 3 and vascular endothelial growth factor thus blocks the blood vessel formation (46).

LncRNA EPR regulates cell proliferation and levels of the mesenchymal and epithelial markers in breast cancer, which is independent of its coding polypeptide. A series of experiments has proven the limitation of EPR gene expression alterations caused by the translated peptide. Therefore, subsequent studies still focus on the lncRNA itself in spite of the coding function of EPR. Upregulated EPR can induce cancer cell apoptosis through regulating cyclin-dependent kinase inhibitor, epithelial cell transforming growth factor $\beta$, and cell cycle arrest (34).

\section{Melanoma}

The polypeptide MELOE-3 produced by the lncRNA meloe is poorly immunogenic and its translation relies on a capdependent mechanism, while two other polypeptides encoded by meloe RNA are less than 50aa in length, with a translation that depends on the IRES, as demonstrated in some melanoma research (53). There is no IRES activity upstream of the MELOE-3-translated ORF, according to in vitro translation and transfection experiments. Furthermore, MELOE-3 is expressed in both melanocytes and melanoma cells, but the other two polypeptides are only expressed in melanoma tumor cells. These two polypeptides' IRES-transactivating factor is specifically activated during the transformation process and that their strong immunogenicity is attributed to their IRES-dependent melanoma specific expression. In brief, these results explain the immune tolerance of the polypeptide MELOE-3 (47).

\section{Others}

A specific ORF Minion encodes a microprotein and gives rise to myoblast fusion mediated by the transmembrane protein Myomaker. This fusion activity is retained in the human homologue, whose transcript is annotated as lncRNA LOC10192972. This discovery may offer a target for inhibiting the oncolytic fusion of cancer cells (54). LINC00116 is considered as a cervical cancer carcinogen (55), but the overexpression of its coding microprotein Mtln induces the conversion of glycolysis to oxidative phosphorylation in cervical cancer (36). This is distinct from the Warburg effect, which suggests that the metabolism increase in cancer cells is associated with glycolysis instead of oxidative phosphorylation in mitochondria (56). Additionally, the great majority of the lncRNAs covered in our review function in cancer. For instance, LINC01420 can promote the proliferation of nasopharyngeal and pancreatic cancer cells, and it also encodes the polypeptide $\operatorname{NoBody}(57,58)$. In addition, LINC00961 translates the mTORC1 inhibitory peptide and induces the apoptosis of melanoma and oral squamous cell carcinoma $(59,60)$. However, the cancer functions of their coding products are still unclear and need further research.

\section{The Function of Long Non-Coding RNA- Encoded Peptides in Other Diseases}

Small peptides have become a potential autoimmune particular treatment in the research progress of autoinflammatory disorder. Thus, to find a peptide without off-target stimulation, Niu et al. preferred to focus on human lncRNA MIR155HG which highly expressed in dendritic cell under inflammation, and subsequently proved that miPEP155 (P155) is the MIR155HG-encoded product. P155 can destroy the function of heat shock cognate protein 70 (HSC70), and then regulate antigen transport. Although the P155 sequence was non-existent in mice, results showed the same things as expected, due to the homology HSC70. In addition, imiquimodinduced mouse model research showed that P155 can act on T cells by skewing their polarization states rather than directly monitoring (48). It was reported that the ORF of IncRNA Aw112010 translated an 84 aa protein, which generated an innate immune response against infection and inflammation in the inflammatory bowel disease mice models (49). Interestingly, using a pancreatic differentiation system, Bjoern et al. found that lncRNA LINC00261 was necessary for pancreatic endocrine cell development. The lack of LINC00261 will seriously reduce insulin production. Then, they singly mutated seven ORFs in turn and the results showed that neither LINC00261' ORFs nor their microproteins were involved in the endocrine function of lncRNA itself. Deleting all ORFs simultaneously also would not reduce the stability of LINC00261 transcript (61). It suggested that LINC00261, not its coding peptide, played a practical role in pancreatic endocrine cell development. The same phenomenon also occurs in the lncRNA EPR in breast cancer. These results remind us to identify the function of lncRNA itself or the coding peptide.

\section{Computational Methods and Data Resources for Predicting Long Non- Coding RNA-Encoded Peptides}

In recent years, with the development of high-throughput sequencing, computational methods for predicting lncRNAencoded proteins have been continuously proposed. In 2009, a ribosome profiling strategy was presented based on the deep sequencing of ribosome-protected mRNA fragments (RPFs) with a length of $\sim 30 \mathrm{nt}$, which could help distinguish "translated RNAs" (62). Wang et al. has reviewed many computational resources for ribosome profiling data processing and interpreting (63). Here, we summarized computational methods through focusing on different key processes of the translation biology based on ribosome profiling data. 


\section{Challenges for Identifying Ribosome-Protected Messenger RNA Fragments}

It is challenging that some RPFs may be mixed with contaminating reads due to technology limitations, such as those derived from ribosomal RNAs (rRNAs). The contaminating reads usually have different length distributions from those real RPFs. This is a key feature to separate bona fide RPFs from contaminating reads such as implemented in FLOSS (64). Rfoot is also designed specifically to distinguish RPFs from other RNA fragments protected by nonribosome RBPs (65). Meanwhile, true RPFs have shown a threenucleotide periodicity because of frame preference. For example, a small wavelet transformation method was employed to denoise RPF reads in RiboWave (66). RiboTaper quantifies the significance of periodic ribo-seq reads via spectral analysis methods (67).

Identifying the ribosome $\mathrm{P}$-site codon for each read is also a challenge because of the different length distribution of RPFs. Some methods used a fixed offset for reads of different length, such as ORFscore (68). However, the simple handling easily caused erroneous assignment of P-sites. To solve this problem, on the one hand, some tools inferred the P-site positions of RPFs based on the offset of the 5' ends of RPFs at start or stop codons as well as the consistency of offsets between reads of different lengths such as RiboProfiling and RiboWaltz $(69,70)$. On the other hand, Scikit-ribo used a random forest classifier considering many features of RPFs including start codons, terminal nucleotides, flanking nucleotides and so on (71).

\section{Methods for Identifying "Translated Open Reading Frames"}

ORF is usually necessary for translation. In the early stage, computational tool for identifying ORFs only considered start codon and stop codon of genome sequences, such as ORFinder (72). However, some RNAs with ORFs still cannot be translated into proteins or peptides. In order to get a higher accuracy, many tools identified novel ORFs based on more translation features in ribosome profiling data. For example, RiboCode identified canonical and non-canonical ORFs and the associated start codons based on the three-nucleotide periodicity of RPFs (73). RiboHMM used a hidden Markov model (HMM) to identify translated ORFs by leveraging the total abundance and the codon periodicity in RPFs (74). Ribosome profiling with Bayesian predictions (RP-BP) used an unsupervised Bayesian approach to predict translated ORFs through an automatic Bayesian periodic fragment length and ribosome P-site offset Selection (BPPS) (75).

\section{Methods for Estimating Translational Efficiency}

Translational efficiency (TE) is a key index which estimates translational regulation. Accurate estimation of TE could help predict "translated RNAs." Most of the methods calculated TE of the ORF as the ratio of reads per kilobase per million mapped reads (RPKM) in ribosome profiling versus that in RNA-seq such as RiboProfiling and Plastid $(70,76)$. However, elongation rate of ribosomes on different ORFs might be different. This would cause errors for estimating TE. To overcome this shortening, Scikit-ribo estimated TE by considering the impact of elongation rate of ribosomes on different codons as well as RNA secondary structures (71). Furthermore, there are some methods focusing on identifying changes in TE under different conditions by modeling the RPF and RNA read counts with some distributions and estimating the significance of TE changes. For example, Xtail used negative binomial distributions (77). Riborex and RiboDiff aimed to run faster by employing a generalized linear model $(78,79)$.

\section{Identification of "Translated RNAs" by MS and Global Translation Initiation Sequencing}

MS and global translation initiation sequencing (GTI-seq) have also been incorporated to identify "translated RNAs." Calviello et al. used ribosome profiling data to make prediction by RiboTaper and used MS spectra to make validation (67). FSPP directly used the overlap of detected small ORF-encoded peptides (SEPs) from ribosome profiling data and MS spectra as target objects (80). GTI-seq could help identify "translated RNAs" by distinguishing ribosome initiation and elongation using two translation inhibitors, lactimidomycin (LTM), and cycloheximide (CHX). Because of combination of ribosome profiling and two different translation-inhibiting chemicals, GTI-seq generates two types of ribosome profiling signal landscapes and improves the identification accuracy of the translation initiation site (81).

\section{Databases for Long Non-Coding RNA-Encoded Peptides}

Currently, there are several databases containing information about lncRNA-encoded peptides. sORFs.org database provided the coding potential small ORFs identified by ribosome profiling (82). Smprot database recorded small peptides predicted by ribosome profiling data and MS spectra (83). Although numerous ncRNA-encoded peptides have been predicted, however, very few were validated by low-throughput experiment. We also developed a database, ncEP, to collect low-throughput experimentally validated ncRNA-encoded peptides from published papers (84). ncEP also contained an online genome browser showing the genome locations of ncRNAs and proteins or peptides as well as their species conservations. More recently, FuncPEP collected experimentally validated and functionally characterized ncRNA-encoded peptides (85). These resources could enrich the knowledge for translation process. The computational methods and databases for lncRNAencoded peptides were summarized in Table 2.

\section{DISCUSSION}

Recent years, more and more studies suggest that some lncRNAs can encode peptides. Most of them are independent of the lncRNA and play important roles in various biological processes. Some lncRNA-encoded peptides may involve in apoptosis inducing and antigen presentation related to autophagy, which implies a close connection with programmed cell death. Furthermore, present coding researches of cancer mainly focus on digestive system, immune system, and skin. Although there is still a vast space to explore, it is no doubt that these peptides will represent new targets for cancer prevention or biomarkers for predicting prognosis of cancer patients. 
TABLE 2 | Computational methods and databases for long non-coding RNA (IncRNA)-encoded peptides.

\begin{tabular}{|c|c|c|c|c|}
\hline $\begin{array}{l}\text { Methods or } \\
\text { databases }\end{array}$ & Description & $\begin{array}{c}\text { Running } \\
\text { environment }\end{array}$ & URL & Reference \\
\hline FLOSS & $\begin{array}{c}\text { Algorithm for ORF identification based on characteristic read length distribution } \\
\text { of true RPFs. }\end{array}$ & $\mathrm{R}$ & NA & $(64)$ \\
\hline Rfoot & $\begin{array}{l}\text { Computational tool for distinguish RPFs from other RNA fragments protected } \\
\text { by non-ribosome RBPs and discovering novel non-coding functions of RNAs. }\end{array}$ & Perl & https://github.com/zhejilab/Rfoot & (65) \\
\hline RiboWave & $\begin{array}{l}\text { Command line tool for denoising RPFs based on wavelet transform and ORF } \\
\text { identification and quantification. }\end{array}$ & $\mathrm{R}$ & https://lulab.github.io/Ribowave & $(66)$ \\
\hline ORFscore & $\begin{array}{l}\text { Algorithm for ORF identification based on quantifying the biased distribution of } \\
\text { RPFs }\end{array}$ & $\mathrm{R}$ & NA & $(68)$ \\
\hline RiboTaper & $\begin{array}{l}\text { Pipeline for ORF identification based on periodic footprint profiles using the } \\
\text { multitaper approach. }\end{array}$ & $\mathrm{R}$ & $\begin{array}{l}\text { https://ohlerlab.mdcberlin.de/software/ } \\
\text { RiboTaper_126/ }\end{array}$ & $(67)$ \\
\hline RiboWaltz & R package for calculating optimal P-site offsets of RPFs. & $\mathrm{R}$ & https://github.com/ & (69) \\
\hline RiboProfiling & $\begin{array}{l}\text { R package for transcript quantification and identification of single- or multi- } \\
\text { amino acids motifs of RPF accumulation. }\end{array}$ & $\mathrm{R}$ & $\begin{array}{c}\text { LabTranslationalArchitectomics/RiboWaltz } \\
\text { http://bioconductor.org/packages/ } \\
\text { RiboProfiling/ }\end{array}$ & $(70)$ \\
\hline Scikit-ribo & $\begin{array}{c}\text { Python package for A-site prediction and robust TE quantification with random } \\
\text { forest classifier model. }\end{array}$ & Python & https://github.com/schatzlab/scikit-ribo & $(71)$ \\
\hline ORFinder & Web server for identifying ORFs. & Web server & https://www.ncbi.nlm.nih.gov/orffinder/ & $(72)$ \\
\hline RiboCode & $\begin{array}{c}\text { Python-based command line tool for de novo identification of ORFs based on } \\
\text { the three-nucleotide periodicity of RPFs. }\end{array}$ & Python & https://github.com/xryanglab/RiboCode & (73) \\
\hline RiboHMM & $\begin{array}{l}\text { Hidden Markov model for ORF identification relying on three nucleotide } \\
\text { periodicity and coverage of RPFs. }\end{array}$ & Python & https://github.com/rajanil/riboHMM & $(74)$ \\
\hline RP-BP & Unsupervised Bayesian pipeline for ORF identification. & Python & https://github.com/dieterich-lab/rp-bp & $(75)$ \\
\hline Plastid & $\begin{array}{l}\text { Python library for nucleotide-resolution analysis of ribosome profiling and TE } \\
\text { estimation. }\end{array}$ & Python & https://github.com/joshuagryphon/plastid & (76) \\
\hline Xtail & $\begin{array}{l}\text { R package for detecting differentially translated ORFs by simulating read } \\
\text { counts with negative binomial distribution. }\end{array}$ & $\mathrm{R}$ & https://github.com/xryanglab/xtail & $(77)$ \\
\hline Riborex & $\begin{array}{l}\text { Fast and flexible R package for calculating genome-wide differences in TE } \\
\text { using DESeq2 and EdgeR. }\end{array}$ & $\mathrm{R}$ & https://github.com/smithlabcode/riborex & $(78)$ \\
\hline RiboDiff & $\begin{array}{l}\text { Pipeline for differential translation analysis based on generalized linear model of } \\
\text { read counts. }\end{array}$ & Python & https://github.com/ratschlab/RiboDiff & (79) \\
\hline FSPP & $\begin{array}{l}\text { Pipeline for identification of small ORF-encoded peptides (SEPs) by combining } \\
\text { ribosome profiling data with MS spectra. }\end{array}$ & $\mathrm{R}$ & https://www.bioinfo.org/FSPP & (80) \\
\hline sORFs.org & $\begin{array}{l}\text { Database for providing the coding potential small ORFs identified by ribosome } \\
\text { profiling. }\end{array}$ & Web server & http://www.sorfs.org & $(82)$ \\
\hline Smprot & $\begin{array}{l}\text { Database for recording small peptides predicted by mass spectrometry (MS) } \\
\text { and ribosome profiling data. }\end{array}$ & Web server & http://bioinfo.ibp.ac.cn/SmProt/ & (83) \\
\hline ncEP & $\begin{array}{l}\text { Database for collecting low-throughput experimentally validated ncRNA- } \\
\text { encoded peptides from published papers. }\end{array}$ & Web server & http://www.jianglab.cn/ncEP/ & (84) \\
\hline ncPEP & $\begin{array}{l}\text { Database for collecting experimentally validated and functionally characterized } \\
\text { ncRNA-encoded peptides. }\end{array}$ & Web server & $\begin{array}{l}\text { https://bioinformatics.mdanderson.org/ } \\
\text { Supplements/FuncPEP/ }\end{array}$ & (85) \\
\hline
\end{tabular}

Here, we described the recent developments of lncRNAencoded peptides in mammals, including the regulatory pathways, functions, computational predicting methods, and data resources. Since humans have more connections and similarities with other mammals, this review provided more convincing information for researchers focusing on the association between lncRNAs and human diseases. Besides, all lncRNA-encoded peptides with oncogene function are provided as far as we know. Such articles have been published increasingly in recent years and need to be unscrambled systematically. This investigation also carries on the computational methods and data resources for key challenges in the translation process and technology development. The computational methods cover from RPF identification to translated ORF identification and TE estimation. According to the technology development, computational methods are mainly based on ribosome profiling, MS, and GTI-seq. Also, lncRNA encoded peptide-related data resources have been summarized including sORFs.org, Smprot and ncEP databases. Different methods and databases focus on different aspects in the translation process. This summing-up could help researchers to choose the suitable computational methods and databases for their specific interests and give new insights for lncRNA translation.

The progress of identifying coding peptides can refer to the following points. First, bioinformatics technology is used to search for lncRNAs with potential coding ability, especially those related to ribosomes. And it is necessary to mutate each ORF to find the practical one. Second, after proving the generation of the coding peptide, determining endogenously expression or localization is an indispensable step to ensure the peptide is translated from the IncRNA instead of other mRNAs. Then, can the translation be stimulated by eIF4E to enhance or reduce the expression of peptide? After that, it is important to confirm whether lncRNA, its coding peptide, or both have functions. And pull-down assay or immunoprecipitation needs be performed to predict the potential interacting partners combined with MS. Last, consider if the lncRNA with translation should be redefined as a protein coding 
gene and think about the classification of coding peptide, including micropeptide, polypeptide, and protein.

In the near future, researches about functions of lncRNAencoded peptides may be a hotpot topic and the coding mechanisms need to be explored in depth. However, there are still some challenges for the further researches. For example, how to predict the coding potential of lncRNA more effectively? Are these IncRNA-encoded peptides different with other peptides? Whether they can be used for diagnosis and prediction of diseases? Whether they can be used as new therapeutic targets or combined with other traditional therapy to improve the curative effects? All of these problems are urgent to be resolved. Interestingly, most of the lncRNA-encoded products in this review are shorter than 100aa. It is difficult to identify long coding proteins with complex biological significance. This may be due to the distribution and the different size of ORFs from IncRNAs and mRNAs. LncRNAs usually harbor ORFs shorter than that of mRNAs. Finally, with the development of new coding prediction tools and genomics technologies, a basis for novel cancer therapeutics should be provided through the

\section{REFERENCES}

1. Rion N, Ruegg MA. LncRNA-encoded peptides: More than translational noise? Cell Res (2017) 27:604-5. doi: 10.1038/cr.2017.35

2. Yin X, Jing Y, Xu H. Mining for missed sORF-encoded peptides. Expert Rev Proteomics (2019) 16:257-66. doi: 10.1080/14789450.2019.1571919

3. Lauressergues D, Couzigou JM, Clemente HS, Martinez Y, Dunand C, Becard G, et al. Primary transcripts of microRNAs encode regulatory peptides. Nature (2015) 520:90-3. doi: 10.1038/nature14346

4. Kong S, Tao M, Shen X, Ju S. Translatable circRNAs and lncRNAs: Driving mechanisms and functions of their translation products. Cancer Lett (2020) 483:59-65. doi: 10.1016/j.canlet.2020.04.006

5. Zhang M, Huang N, Yang X, Luo J, Yan S, Xiao F, et al. A novel protein encoded by the circular form of the SHPRH gene suppresses glioma tumorigenesis. Oncogene (2018) 37:1805-14. doi: 10.1038/s41388-017-0019-9

6. Zheng X, Chen L, Zhou Y, Wang Q, Zheng Z, Xu B, et al. A novel protein encoded by a circular RNA circPPP1R12A promotes tumor pathogenesis and metastasis of colon cancer via Hippo-YAP signaling. Mol Cancer (2019) 18:47. doi: 10.1186/s12943-019-1010-6

7. Zhang M, Zhao K, Xu X, Yang Y, Yan S, Wei P, et al. A peptide encoded by circular form of LINC-PINT suppresses oncogenic transcriptional elongation in glioblastoma. Nat Commun (2018) 9:4475. doi: 10.1038/s41467-01806862-2

8. Bhan A, Soleimani M, Mandal SS. Long noncoding RNA and cancer: A new paradigm. Cancer Res (2017) 77:3965-81. doi: 10.1158/0008-5472.CAN-16-2634

9. Arun G, Diermeier SD, Spector DL. Therapeutic targeting of long non-coding RNAs in cancer. Trends Mol Med (2018) 24:257-77. doi: 10.1016/ j.molmed.2018.01.001

10. Batista PJ, Chang HY. Long noncoding RNAs: cellular address codes in development and disease. Cell (2013) 152:1298-307. doi: 10.1016/j.cell.2013.02.012

11. Choi SW, Kim HW, Nam JW. The small peptide world in long noncoding RNAs. Brief Bioinform (2019) 20:1853-64. doi: 10.1093/bib/bby055

12. Min K, Davila S, Zealy RW, Lloyd LT, Lee IY, Lee R, et al. eIF4E phosphorylation by MST1 reduces translation of a subset of mRNAs, but increases lncRNA translation. Biochim Biophys Acta (BBA) - Gene Regul Mech (2017) 1860:761-72. doi: 10.1016/j.bbagrm.2017.05.002

13. Szafron LM, Balcerak A, Grzybowska EA, Pienkowska-Grela B, FelisiakGolabek A, Podgorska A, et al. The novel gene CRNDE encodes a nuclear peptide (CRNDEP) which is overexpressed in highly proliferating tissues. PLoS One (2015) 10:e0127475. doi: 10.1371/journal.pone.0127475

14. Frank MJ, Smith LG. A small, novel protein highly conserved in plants and animals promotes the polarized growth and division of maize leaf epidermal cells. Curr Biol (2002) 12:849-53. doi: 10.1016/s0960-9822(02)00819-9 exploration of special translation mechanisms and the functions of the coding products.

\section{AUTHOR CONTRIBUTIONS}

LW and WJ designed the study and drafted the manuscript. JX and HL summarized the theories and drafted the manuscript. All authors contributed to the article and approved the submitted version.

\section{FUNDING}

This work was supported by grants from the Fundamental Research Funds for the Central Universities (Grant No. 2242020K40131) and the National Natural Science Foundation of China (Grant No. 81972478).

15. Röhrig H, Schmidt J, Miklashevichs E, Schell J, John M. Soybean ENOD40 encodes two peptides that bind to sucrose synthase. Proc Natl Acad Sci - PNAS (2002) 99:1915-20. doi: 10.1073/pnas.022664799

16. Pauli A, Norris ML, Valen E, Chew GL, Gagnon JA, Zimmerman S, et al. Toddler: An embryonic signal that promotes cell movement via apelin receptors. Science (2014) 343:1248636-1248636. doi: 10.1126/science.1248636

17. Matsumoto A, Nakayama KI. Hidden peptides encoded by putative noncoding RNAs. Cell Struct Funct (2018) 43:75-83. doi: 10.1247/csf.18005

18. Zhu S, Wang J, He Y, Meng N, Yan GR. Peptides/Proteins encoded by noncoding RNA: A novel resource bank for drug targets and biomarkers. Front Pharmacol (2018) 9:1295. doi: 10.3389/fphar.2018.01295

19. Wu P, Mo Y, Peng M, Tang T, Zhong Y, Deng X, et al. Emerging role of tumor-related functional peptides encoded by lncRNA and circRNA. Mol Cancer (2020) 19:22. doi: 10.1186/s12943-020-1147-3

20. Abu-Remaileh M, Wyant GA, Kim C, Laqtom NN, Abbasi M, Chan SH, et al. Lysosomal metabolomics reveals V-ATPase- and mTOR-dependent regulation of amino acid efflux from lysosomes. Science (2017) 358:807-13. doi: 10.1126/science.aan6298

21. Zoncu R, Bar-Peled L, Efeyan A, Wang S, Sancak Y, Sabatini DM. mTORC1 senses lysosomal amino acids through an inside-out mechanism that requires the vacuolar H(+)-ATPase. Science (2011) 334:678-83. doi: 10.1126/science.1207056

22. Matsumoto A, Pasut A, Matsumoto M, Yamashita R, Fung J, Monteleone E, et al. mTORC1 and muscle regeneration are regulated by the LINC00961-encoded SPAR polypeptide. Nature (2017) 541:228-32. doi: 10.1038/nature21034

23. Baraldo M, Geremia A, Pirazzini M, Nogara L, Solagna F, Türk C, et al. Skeletal muscle mTORC1 regulates neuromuscular junction stability. J Cachexia Sarcopenia Muscle (2019) 11:208-25. doi: 10.1002/jcsm.12496

24. Spencer HL, Sanders R, Boulberdaa M, Meloni M, Cochrane A, Spiroski A, et al. The LINC00961 transcript and its encoded micropeptide, small regulatory polypeptide of amino acid response, regulate endothelial cell function. Cardiovasc Res (2020) 116:1981-94. doi: 10.1093/cvr/cvaa008

25. Wang L, Fan J, Han L, Qi H, Wang Y, Wang H, et al. The micropeptide LEMP plays an evolutionarily conserved role in myogenesis. Cell Death Dis (2020) 11:357. doi: 10.1038/s41419-020-2570-5

26. MacLennan DH, Asahi M, Tupling AR. The regulation of SERCA-type pumps by phospholamban and sarcolipin. Ann N Y Acad Sci (2003) 986:472-80. doi: 10.1111/j.1749-6632.2003.tb07231.x

27. Anderson DM, Anderson KM, Chang CL, Makarewich CA, Nelson BR, McAnally JR, et al. A micropeptide encoded by a putative long noncoding RNA regulates muscle performance. Cell (2015) 160:595-606. doi: 10.1016/ j.cell.2015.01.009

28. Nelson BR, Makarewich CA, Anderson DM, Winders BR, Troupes CD, Wu F, et al. A peptide encoded by a transcript annotated as long noncoding RNA 
enhances SERCA activity in muscle. Science (2016) 351:271-5. doi: 10.1126/ science.aad 4076

29. Magny EG, Pueyo JI, Pearl FMG, Cespedes MA, Niven JE, Bishop SA, et al. Conserved regulation of cardiac calcium uptake by peptides encoded in small open reading frames. Science (2013) 341:1113-6. doi: 10.1126/science.1238802

30. Kurosaki T, Maquat LE. Nonsense-mediated mRNA decay in humans at a glance. J Cell Sci (2016) 129:461-7. doi: 10.1242/jcs.181008

31. D'Lima NG, Ma J, Winkler L, Chu Q, Loh KH, Corpuz EO, et al. A human microprotein that interacts with the mRNA decapping complex. Nat Chem Biol (2017) 13:174-80. doi: 10.1038/nchembio.2249

32. Wery M, Descrimes M, Vogt N, Dallongeville AS, Gautheret D, Morillon A. Nonsense-mediated decay restricts lncRNA levels in yeast unless blocked by double-stranded RNA structure. Mol Cell (2016) 61:379-92. doi: 10.1016/ j.molcel.2015.12.020

33. Hartford CCR, Lal A. When long noncoding becomes protein coding. Mol Cell Biol (2020) 40:e00528-19. doi: 10.1128/MCB.00528-19

34. Rossi M, Bucci G, Rizzotto D, Bordo D, Marzi MJ, Puppo M, et al. LncRNA EPR controls epithelial proliferation by coordinating Cdkn1a transcription and mRNA decay response to TGF- $\beta$. Nat Commun (2019) 10:1969. doi: 10.1038/s41467-019-09754-1

35. Ren M, Phoon CKL, Schlame M. Metabolism and function of mitochondrial cardiolipin. Prog Lipid Res (2014) 55:1-16. doi: 10.1016/j.plipres.2014.04.001

36. Stein CS, Jadiya P, Zhang X, McLendon JM, Abouassaly GM, Witmer NH, et al. Mitoregulin: A lncRNA-encoded microprotein that supports mitochondrial supercomplexes and respiratory efficiency. Cell Rep (2018) 23:3710-20.e8. doi: 10.1016/j.celrep.2018.06.002

37. Makarewich CA, Baskin KK, Munir AZ, Bezprozvannaya S, Sharma G, Khemtong C, et al. MOXI is a mitochondrial micropeptide that enhances fatty acid B-Oxidation. Cell Rep (2018) 23:3701-9. doi: 10.1016/j.celrep.2018.05.058

38. Chugunova A, Loseva E, Mazin P, Mitina A, Navalayeu T, Bilan D, et al. LINC00116 codes for a mitochondrial peptide linking respiration and lipid metabolism. Proc Natl Acad Sci (2019) 116:4940-5. doi: 10.1073/pnas.1809105116

39. Huang J, Chen M, Chen D, Gao X, Zhu S, Huang H, et al. A peptide encoded by a putative lncRNA HOXB-AS3 suppresses colon cancer growth. Mol Cell (2017) 68:171-84.e6. doi: 10.1016/j.molcel.2017.09.015

40. Meng N, Chen M, Chen D, Chen XH, Wang JZ, Zhu S, et al. Small protein hidden in lncRNA LOC90024 promotes "cancerous" RNA splicing tumorigenesis. Adv Sci (2020) 7:1903233. doi: 10.1002/advs.201903233

41. Zhu S, Wang J, Chen D, He Y, Meng N, Chen M, et al. An oncopeptide regulates $\mathrm{m} 6 \mathrm{~A}$ recognition by the $\mathrm{m} 6 \mathrm{~A}$ reader IGF2BP1 and tumorigenesis. Nat Commun (2020) 11:1685. doi: 10.1038/s41467-020-15403-9

42. Pang Y, Liu Z, Han H, Wang B, Li W, Mao C, et al. Peptide SMIM30 promotes HCC development by inducing SRC/YES1 membrane anchoring and MAPK pathway activation. J Hepatol (2020) 73:1155-69. doi: 10.1016/j.jhep.2020.05.028

43. Lun Y, Pan Z, Liu S, Sun J, Han M, Liu B, et al. The peptide encoded by a novel putative IncRNA HBVPTPAP inducing the apoptosis of hepatocellular carcinoma cells by modulating JAK/STAT signaling pathways. Virus Res (2020) 287:198104. doi: 10.1016/j.virusres.2020.198104

44. Wu S, Zhang L, Deng J, Guo B, Li F, Wang Y, et al. A novel micropeptide encoded by Y-linked LINC00278 links cigarette smoking and AR signaling in male esophageal squamous cell carcinoma. Cancer Res (2020) 88:2790-803. doi: 10.1158/0008-5472.CAN-19-3440

45. Guo B, Wu S, Zhu X, Zhang L, Deng J, Li F, et al. Micropeptide CIP2A-BP encoded by LINC00665 inhibits triple-negative breast cancer progression. EMBO J (2020) 39:e102190-n/a. doi: 10.15252/embj.2019102190

46. Wang Y, Wu S, Zhu X, Zhang L, Deng J, Li F, et al. LncRNA-encoded polypeptide ASRPS inhibits triple-negative breast cancer angiogenesis. J Exp Med (2020) 217:e20190950. doi: 10.1084/jem.20190950

47. Charpentier M, Croyal M, Carbonnelle D, Fortun A, Florenceau L, Rabu C, et al. IRES-dependent translation of the long non coding RNA meloe in melanoma cells produces the most immunogenic MELOE antigens. Oncotarget (2016) 7:59704. doi: 10.18632/oncotarget.10923

48. Niu L, Lou F, Sun Y, Sun L, Cai X, Liu Z, et al. A micropeptide encoded by lncRNA MIR155HG suppresses autoimmune inflammation via modulating antigen presentation. Sci $A d v$ (2020) 6:eaaz2059. doi: 10.1126/sciadv.aaz2059

49. Jackson R, Kroehling L, Khitun A, Bailis W, Jarret A, York AG, et al. The translation of non-canonical open reading frames controls mucosal immunity. Nature (2018) 564:434-8. doi: 10.1038/s41586-018-0794-7
50. Chakraborty S, Andrieux G, Hasan A, Ahmed M, Hosen MI, Rahman T, et al. Harnessing the tissue and plasma IncRNA-peptidome to discover peptidebased cancer biomarkers. Sci Rep (2019) 9:12322. doi: 10.1038/s41598-01948774-1

51. Polycarpou-Schwarz M, Groß M, Mestdagh P, Schott J, Grund SE, Hildenbrand $\mathrm{C}$, et al. The cancer-associated microprotein CASIMO1 controls cell proliferation and interacts with squalene epoxidase modulating lipid droplet formation. Oncogene (2018) 37:4750-68. doi: 10.1038/s41388018-0281-5

52. Papaioannou D, Petri A, Dovey OM, Terreri S, Wang E, Collins FA, et al. The long non-coding RNA HOXB-AS3 regulates ribosomal RNA transcription in NPM1-mutated acute myeloid leukemia. Nat Commun (2019) 10:5351. doi: 10.1038/s41467-019-13259-2

53. Carbonnelle D, Vignard V, Sehedic D, Moreau-Aubry A, Florenceau L, Charpentier M, et al. The melanoma antigens MELOE-1 and MELOE-2 are translated from a bona fide polycistronic mRNA containing functional IRES sequences. PLoS One (2013) 8:e75233. doi: 10.1371/journal.pone.0075233

54. Zhang Q, Vashisht AA, O Rourke J, Corbel SY, Moran R, Romero A, et al. The microprotein Minion controls cell fusion and muscle formation. Nat Commun (2017) 8:15664. doi: $10.1038 /$ ncomms 15664

55. Lai Y, Zhou B, Tan Q, Xu J, Wan T, Zhang L. LINC00116 enhances cervical cancer tumorigenesis through miR-106a/c-Jun pathway. J Cell Biochem (2019) 121:2247-57. doi: 10.1002/jcb.29447

56. Israelsen WJ, Vander HM. Pyruvate kinase: Function, regulation and role in cancer. Semin Cell Dev Biol (2015) 43:43-51. doi: 10.1016/j.semcdb.2015.08.004

57. Yang L, Tang Y, He Y, Wang Y, Lian Y, Xiong F, et al. High Expression of LINC01420 indicates an unfavorable prognosis and modulates cell migration and invasion in nasopharyngeal carcinoma. J Cancer (2017) 8:97-103. doi: $10.7150 /$ jca. 16819

58. Zhai H, Zhang X, Sun X, Zhang D, Ma S. Long non-coding RNA LINC01420 contributes to pancreatic cancer progression through targeting KRAS protooncogene. Dig Dis Sci (2020) 65:1042-52. doi: 10.1007/s10620-019-05829-7

59. Mu X, Mou KH, Ge R, Han D, Zhou Y, Wang LJ. Linc00961 inhibits the proliferation and invasion of skin melanoma by targeting the miR367/PTEN axis. Int J Oncol (2019) 55:708-20. doi: 10.3892/ijo.2019.4848

60. Pan LN, Sun YR. LINC00961 suppresses cell proliferation and induces cell apoptosis in oral squamous cell carcinoma. Eur Rev Med Pharmacol Sci (2019) 23:3358-65. doi: 10.26355/eurrev_201904_17699

61. Gaertner B, van Heesch S, Schneider-Lunitz V, Schulz JF, Witte F, Blachut S, et al. A human ESC-based screen identifies a role for the translated lncRNA LINC00261 in pancreatic endocrine differentiation. eLife (2020) 9:e58659. doi: 10.7554/eLife.58659

62. Ingolia NT, Ghaemmaghami S, Newman JR, Weissman JS. Genome-wide analysis in vivo of translation with nucleotide resolution using ribosome profiling. Science (2009) 324:218-23. doi: 10.1126/science.1168978

63. Wang $\mathrm{H}$, Wang $\mathrm{Y}$, Xie Z. Computational resources for ribosome profiling: from database to Web server and software. Brief Bioinform (2019) 20:144-55. doi: 10.1093/bib/bbx093

64. Ingolia NT, Brar GA, Stern-Ginossar N, Harris MS, Talhouarne GJ, Jackson $\mathrm{SE}$, et al. Ribosome profiling reveals pervasive translation outside of annotated protein-coding genes. Cell Rep (2014) 8:1365-79. doi: 10.1016/j.celrep. 2014.07.045

65. Ji Z. Rfoot: Transcriptome-scale identification of RNA-protein complexes from ribosome profiling data. Curr Protoc Mol Biol (2018) 124:e66. doi: $10.1002 / \mathrm{cpmb} .66$

66. Xu Z, Hu L, Shi B, Geng S, Xu L, Wang D, et al. Ribosome elongating footprints denoised by wavelet transform comprehensively characterize dynamic cellular translation events. Nucleic Acids Res (2018) 46:e109-9. doi: 10.1093/nar/gky533

67. Calviello L, Mukherjee N, Wyler E, Zauber H, Hirsekorn A, Selbach M, et al. Detecting actively translated open reading frames in ribosome profiling data. Nat Methods (2016) 13:165-70. doi: 10.1038/nmeth.3688

68. Bazzini AA, Johnstone TG, Christiano R, Mackowiak SD, Obermayer B, Fleming ES, et al. Identification of small ORFs in vertebrates using ribosome footprinting and evolutionary conservation. EMBO J (2014) 33:981-93. doi: $10.1002 / \mathrm{embj} .201488411$

69. Lauria F, Tebaldi T, Bernabo P, Groen E, Gillingwater TH, Viero G. riboWaltz: Optimization of ribosome P-site positioning in ribosome profiling data. PLoS Comput Biol (2018) 14:e1006169. doi: 10.1371/journal.pcbi.1006169 
70. Ingolia NT. Ribosome profiling: new views of translation, from single codons to genome scale. Nat Rev Genet (2014) 15:205-13. doi: 10.1038/nrg3645

71. Fang H, Huang YF, Radhakrishnan A, Siepel A, Lyon GJ, Schatz MC. Scikit-ribo enables accurate estimation and robust modeling of translation dynamics at codon resolution. Cell Syst (2018) 6:180-91.e4. doi: 10.1016/j.cels.2017.12.007

72. Rombel IT, Sykes KF, Rayner S, Johnston SA. ORF-FINDER: a vector for high-throughput gene identification. Gene (2002) 282:33-41. doi: 10.1016/ s0378-1119(01)00819-8

73. Xiao Z, Huang R, Xing X, Chen Y, Deng H, Yang X. De novo annotation and characterization of the translatome with ribosome profiling data. Nucleic Acids Res (2018) 46:e61. doi: 10.1093/nar/gky179

74. Raj A, Wang SH, Shim H, Harpak A, Li YI, Engelmann B, et al. Thousands of novel translated open reading frames in humans inferred by ribosome footprint profiling. eLife (2016) 5:e13328. doi: 10.7554/eLife.13328

75. Malone B, Atanassov I, Aeschimann F, Li X, Grosshans H, Dieterich C. Bayesian prediction of RNA translation from ribosome profiling. Nucleic Acids Res (2017) 45:2960-72. doi: 10.1093/nar/gkw1350

76. Dunn JG, Weissman JS. Plastid: nucleotide-resolution analysis of nextgeneration sequencing and genomics data. BMC Genomics (2016) 17:958. doi: 10.1186/s12864-016-3278-x

77. Xiao Z, Zou Q, Liu Y, Yang X. Genome-wide assessment of differential translations with ribosome profiling data. Nat Commun (2016) 7:11194. doi: 10.1038/ncomms11194

78. Li W, Wang W, Uren PJ, Penalva L, Smith AD. Riborex: fast and flexible identification of differential translation from Ribo-seq data. Bioinformatics (2017) 33:1735-7. doi: 10.1093/bioinformatics/btx047

79. Zhong Y, Karaletsos T, Drewe P, Sreedharan VT, Kuo D, Singh K, et al. RiboDiff: detecting changes of mRNA translation efficiency from ribosome footprints. Bioinformatics (2017) 33:139-41. doi: 10.1093/bioinformatics/btw585
80. Li H, Xiao L, Zhang L, Wu J, Wei B, Sun N, et al. FSPP: A tool for genomewide prediction of smORF-encoded peptides and their functions. Front Genet (2018) 9:96. doi: 10.3389/fgene.2018.00096

81. Wan J, Qian SB. TISdb: a database for alternative translation initiation in mammalian cells. Nucleic Acids Res (2014) 42:D845-50. doi: 10.1093/nar/ gkt1085

82. Olexiouk V, Crappe J, Verbruggen S, Verhegen K, Martens L, Menschaert G. sORFs.org: a repository of small ORFs identified by ribosome profiling. Nucleic Acids Res (2016) 44:D324-9. doi: 10.1093/nar/gkv1175

83. Hao Y, Zhang L, Niu Y, Cai T, Luo J, He S, et al. SmProt: a database of small proteins encoded by annotated coding and non-coding RNA loci. Brief Bioinform (2018) 19:636-43. doi: 10.1093/bib/bbx005

84. Liu H, Zhou X, Yuan M, Zhou S, Huang YE, Hou F, et al. ncEP: A manually curated database for experimentally validated ncRNA-encoded proteins or peptides. J Mol Biol (2020) 432:3364-8. doi: 10.1016/j.jmb.2020.02.022

85. Dragomir MP, Manyam GC, Ott LF, Berland L, Knutsen E, Ivan C, et al. FuncPEP: A database of functional peptides encoded by non-coding RNAs. Noncoding RNA (2020) 6:41. doi: 10.3390/ncrna6040041

Conflict of Interest: The authors declare that the research was conducted in the absence of any commercial or financial relationships that could be construed as a potential conflict of interest.

Copyright (c) 2021 Xing, Liu, Jiang and Wang. This is an open-access article distributed under the terms of the Creative Commons Attribution License (CC BY). The use, distribution or reproduction in other forums is permitted, provided the original author(s) and the copyright owner(s) are credited and that the original publication in this journal is cited, in accordance with accepted academic practice. No use, distribution or reproduction is permitted which does not comply with these terms. 